\title{
PRE-IMAGES OF BOUNDARY POINTS OF THE NUMERICAL RANGE
}

\author{
Timothy LEAKe, BRIAN Lins AND ILYA M. SPITKOVSKY
}

\begin{abstract}
This paper considers matrices $A \in M_{n}(\mathbb{C})$ whose numerical range contains boundary points generated by multiple linearly independent vectors. Sharp bounds for the maximum number of such boundary points (excluding flat portions) are given for unitarily irreducible matrices of dimension $\leqslant 5$. An example is provided to show that there may be infinitely many for $n=6$. For matrices unitarily similar to tridiagonal, however, a finite upper bound is found for all $n$. A somewhat unexpected byproduct of this is an explicit example of $A \in M_{5}(\mathbb{C})$ which is not tridiagonalizable via a unitary similarity.
\end{abstract}

Mathematics subject classification (2010): Primary 15A60, 47A12; Secondary 54C08. Keywords and phrases: Field of values, numerical range, inverse continuity, weak continuity.

\section{REFERENCES}

[1] H. BAumgäRTEl, Analytic perturbation theory for matrices and operators, Operator theory, Birkhäuser Verlag, 1985.

[2] E. BROWN AND I. SPITKOVSKY, On flat portions on the boundary of the numerical range, Linear Algebra Appl., 390: 75-109, 2004.

[3] R. CARDEN, A simple algorithm for the inverse field of values problem, Inverse Problems, 25 (11): $115019,9,2009$.

[4] M.-D. ChOI, D. W. KRIBS, AND K. ŻYCZKOWS KI, Higher-rank numerical ranges and compression problems, Linear Algebra Appl., 418 (2-3): 828-839, 2006.

[5] D. Corey, C. Johnson, R. Kirk, B. Lins, And I. M. Spitkovsky, Continuity properties of vectors realizing points in the classical field of values, Linear Multilinear Algebra, 61: 1329-1338, 2013.

[6] C. K. Fong AND P. Y. Wu, Band-diagonal operators, Linear Algebra Appl., 248: 185-204, 1996.

[7] T. Gallay And D. Serre, Numerical measure of a complex matrix, Communications on Pure and Applied Mathematics, 65 (3): 287-336, 2012.

[8] H.-L. GAU AND P. Y. WU, Line segments and elliptic arcs on the boundary of a numerical range, Linear Multilinear Algebra, 56 (1-2): 131-142, 2008.

[9] K. E. Gustafson And D. K. M. RaO, Numerical Range. The Field of Values of Linear Operators and Matrices, Springer, New York, 1997.

[10] R. A. Horn And C. R. Johnson, Topics in Matrix Analysis, Cambridge University Press, Cambridge, 1991.

[11] E. A. Jonckheere, F. Ahmad, And E. Gutkin, Differential topology of numerical range, Linear Algebra Appl., 279 (1-3): 227-254, 1998.

[12] M. Joswig And B. STRAub, On the numerical range map, Journal of the Australian Mathematical Society, 65 (02): 267-283, 1998.

[13] D. Keeler, L. Rodman, And I. Spitkovsky, The numerical range of $3 \times 3$ matrices, Linear Algebra Appl., 252: 115-139, 1997.

[14] R. Kippenhahn, Über den Wertevorrat einer Matrix, Math. Nachr., 6: 193-228, 1951.

[15] C.-K. Li, I. SPITKOVSKY, AND S. SHUKLA, Equality of higher numerical ranges of matrices and a conjecture of Kippenhahn on Hermitian pencils, Linear Algebra Appl., 270: 323-349, 1998.

[16] W. E. LongSTAFF, On tridiagonalization of matrices, Linear Algebra Appl., 109: 153-163, 1988.

[17] T. Moran AND I. M. SpitKovsky, On almost normal matrices, Textos de Matemática, 44: 131$144,2013$. 
[18] V. Pati, Unitary tridiagonalization in $M(4, \mathbb{C})$, Proc. Indian Acad. Sci. Math. Sci., 111 (4): 381-397, 2001.

[19] F. Rellich, Perturbation Theory of Eigenvalue Problems, Notes on Mathematics and its Applications, Taylor \& Francis, 1969.

[20] H. SHAPIRO, A conjecture of Kippenhahn about the characteristic polynomial of a pencil generated by two Hermitian matrices, II, Linear Algebra Appl., 45: 97-108, 1982.

[21] H. SHAPIRO, On a conjecture of Kippenhahn about the characteristic polynomial of a pencil generated by two Hermitian matrices, I, Linear Algebra Appl., 43: 201-221, 1982.

[22] B. STURMFELS, Tridiagonalization of complex matrices and a problem of Longstaff, Linear Algebra Appl., 109: 165-166, 1988.

[23] B.-S. TAM AND S. YANG, On matrices whose numerical ranges have circular or weak circular symmetry, Linear Algebra Appl., 302/303:193-221, 1999, Special issue dedicated to Hans Schneider (Madison, WI, 1998).

[24] N.-K. Tsing, The constrained bilinear form and the C-numerical range, Linear Algebra Appl., 56: 195-206, 1984.

[25] P. Y. WU, Numerical ranges as circular discs, Appl. Math. Lett., 24 (12): 2115-2117, 2011. 